\title{
A framework for predicting the non-visual effects of daylight - Part II: the simulation model
}

J Mardaljevic $\mathrm{PhD}^{a}, \mathrm{M}$ Andersen $\mathrm{PhD}^{b}, \mathrm{~N} \mathrm{Roy}^{c}$ and $\mathrm{J}$ Christoffersen $\mathrm{PhD}^{c}$ ${ }^{a}$ School of Civil and Building Engineering, Loughborough University, Loughborough, UK

${ }^{b}$ Interdisciplinary Laboratory of Performance-Integrated Design, School of Architecture, Civil and Environmental Engineering (ENAC), Ecole Polytechnique Fédérale de Lausanne (EPFL), Lausanne, Switzerland ${ }^{c}$ VELUX A/S, Ådalsvej 99, DK-2970 Hørsholm, Denmark

Address for correspondence: Professor John Mardaljevic, School of Civil and Building Engineering, Loughborough University, Loughborough, Leicestershire, LE11 3TU, UK E-mail: j.mardaljevic@lboro.ac.uk 
This paper describes a climate-based simulation framework devised to investigate the potential for the non-visual effects of daylight in buildings. It is part 2 of a study where the first paper focused on the formulation of the photobiological underpinnings of a thresholdbased model configured for lighting simulation from the perspective of the human nonvisual system (e.g. circadian response). This threshold-based model employs a static dose-response curve and instantaneous exposure of daylight at the eye to estimate the magnitude of the non-visual effect as a first step towards a simulation framework that would establish a link between light exposure at the eye in an architectural context and expected effects on the non-visual system. In addition to being highly sensitive to the timing and duration of light exposure, the non-visual system differs fundamentally from the visual system in its action spectrum. The photosensitivity of the retinal ganglion cells that communicate light exposure to the brain is known to be shifted to the blue with respect to the photopic sensitivity curve. Thus the spectral character of daylight also becomes a sensitive factor in the magnitude of the predicted non-visual effect. This is accounted for in the model by approximating 'yellow' sunlight, 'grey' skylight and 'blue' skylight to three distinct CIE illuminant types, and then tracking their 'circadianlux' weighted contributions in the summation of daylight received at the eye. A means to 'condense' non-visual effects into a synthesised graphical format for the year, split by periods of the day, is described in terms of how such a format could inform design decisions. The sensitivity of the simulation model's predictions to prevailing climate and building orientation is demonstrated by comparing results from eight European locations.

\section{Introduction}

It is now well recognised that illumination received at the eye is responsible for a number of effects on the human body that are unrelated in any direct sense to vision. Light has measurable neuroendocrine and neurobehavioral effects on the human body, in particular with respect to maintaing a regular sleep-wake cycle that is entrained to the natural diurnal cycle of night and day [1]. Additionally there is evidence to suggest links between daylight illumination in particular and alertness, productivity and academic achievement [2]. In the early 1990s it was discovered that the eye possesses an additional non-rod, noncone photoreceptor named photosensitive retinal ganglion cells or pRGC [3]. The pRGC communicate light induced signals to the suprachiasmatic nuclei (sometimes referred to as the 'circadian pacemaker') in the anterior hypothalamus of the brain. This and related discoveries have led the emergence of a new area for photo-biology research concerned with daylight/light exposure and its non-visual effects [4][5][6][7]. There is now sufficient empirical data to begin to formulate investigative simulation models for the non-visual effects caused by exposure to daylight inside buildings, and how these are influenced by the prevailing climate and design parameters such as the size and aspect of glazing elements. An earlier article described in detail the assumptions underlying the static photobiology model developed as a proof-of-concept for this purpose [8]. The focus for this paper is the climate-based simulation model that was devised to predict and visualise the degree and distribution of potential non-visual effect produced by daylight in buildings.

\section{Outline of the climate-based simulation model}

The simulation model devised to investigate the non-visual effects of daylight can be considered as comprising four distinct parts A to D, shown in the schematic given in Figure 1. The four parts of the simulation model are described in the following four sections. 


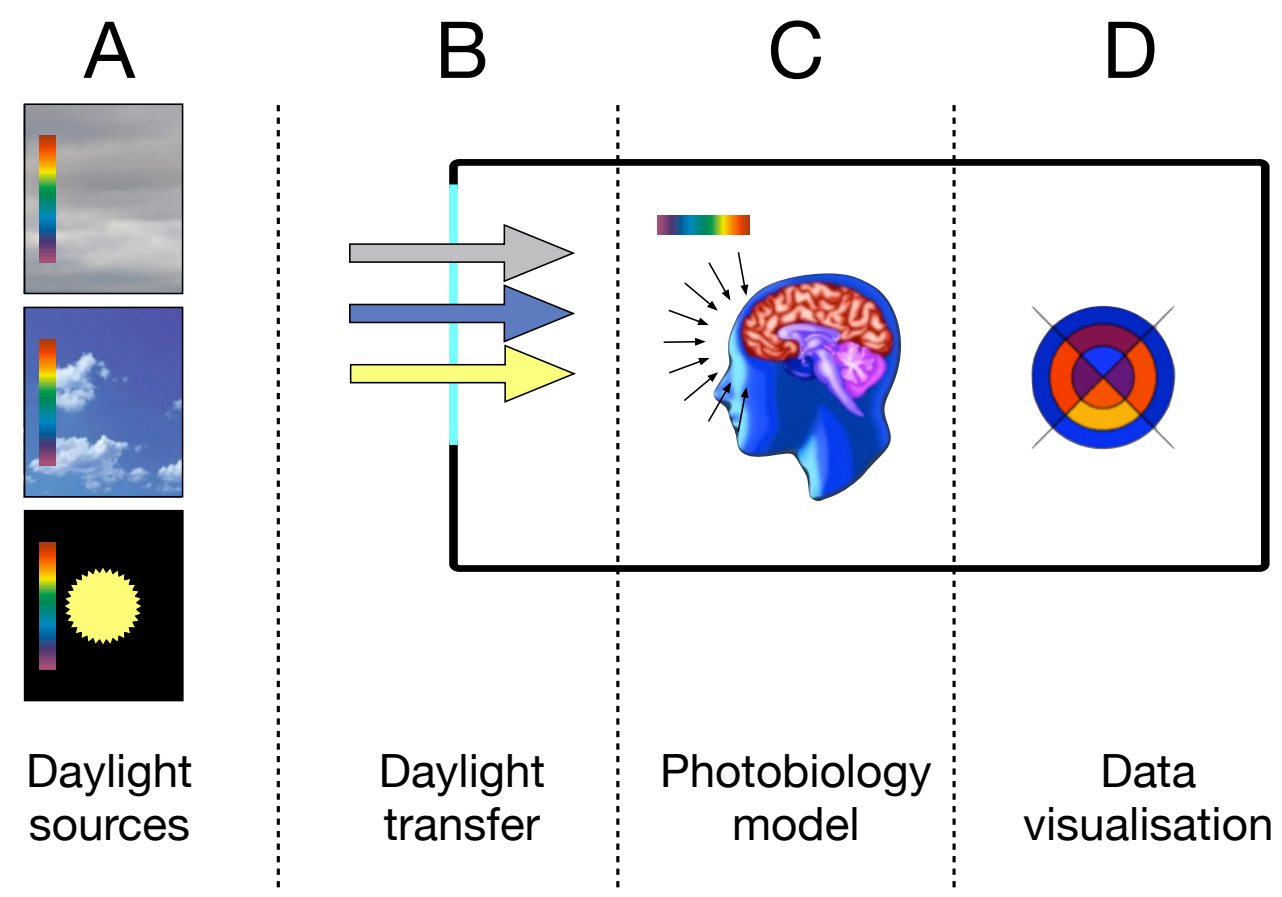

Figure 1: The four parts of a simulation model for non-visual effects (available in colour online)

\subsection{Part A: Sources of daylight}

Given the self-evident nature of the seasonal pattern in daylight availability, a function of both the sun position and the seasonal patterns of cloudiness, any form of daylight evaluation - whether for visual or non-visual effects - should in the first instance be founded on a full year of data. The principal sources of daylight availability for modelling purposes are the standard climate files. These were originally created for use by dynamic thermal modelling programs [9]. These datasets contain averaged hourly values for a full year, i.e. 8,760 values for each parameter. For daylight simulation the required parameters may be either of the following pairs:

- Global horizontal irradiance and either diffuse horizontal irradiance or direct normal irradiance.

- Global horizontal illuminance and either diffuse horizontal illuminance or direct normal illuminance.

Standard climate data for a large number of locales across the world are freely available for download from several websites. One of the most comprehensive repositories is that compiled for use with the EnergyPlus thermal simulation program [10]. Diffuse horizontal and direct normal illuminance data from one of the eight standardised climate files used in the complete study are shown as 'temporal maps' in Figure 2. The 8,760 hourly values were reordered into a 365 (i.e. days) by 24 (i.e. hours) array. The false-colour shading represents the magnitude of the illuminance with zero values shaded light-grey. In the plot for diffuse illuminance the grey area indicates the hours of darkness. Presented in this way it is easy to appreciate both the prevailing patterns in either quantity and their short-term variability. Most obvious are the daily and seasonal patterns for both illuminances: short 
periods of daylight in the winter months, longer in summer. The hour-by-hour variation in the direct normal illuminance is clearly visible, though it is also present to a lesser degree in the diffuse horizontal illuminance (i.e. from the sky). The dashed vertical white lines indicate the start and end of daylight saving times. Note - this and other figures are best viewed in colour on-line.
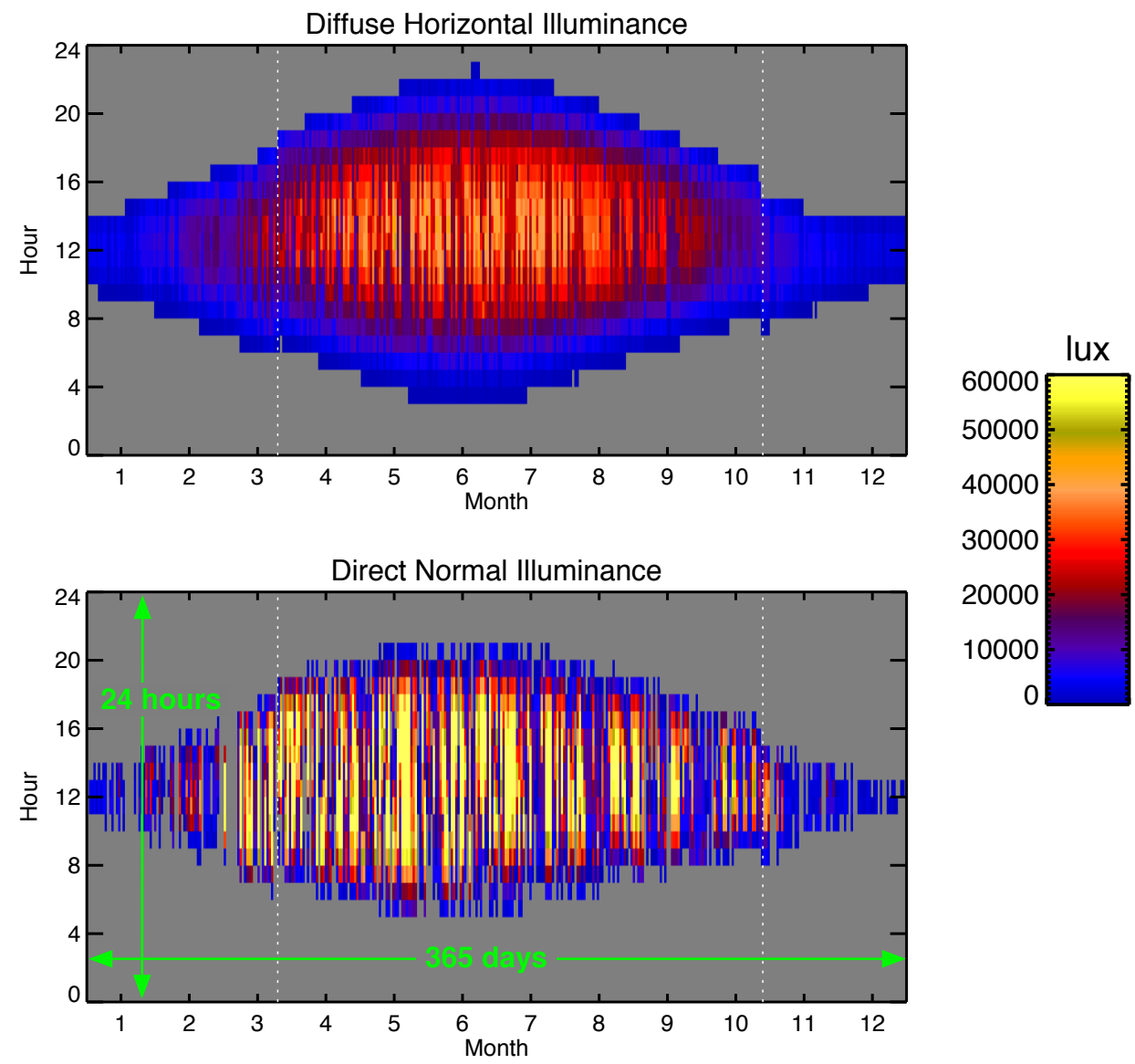

Figure 2: Temporal maps showing the diffuse horizontal and direct normal illuminance data from the standardised climate file for Ostersund, Sweden (available in colour online)

Another consideration for this study was a way to estimate the spectral differences in the daylight according to source, e.g: 'yellow' light from the sun; 'grey' light from an overcast sky; and, 'blue' light from a clear sky. The spectral character of the source illumination is believed to be a factor because the action spectrum for the photosensitive retinal ganglion cells differs from the photopic sensitivity curve (see Section 2.3). If it were the same, we could have used standard photopic units i.e. lux throughout. We concentrated on the spectral properties of the daylight sources rather than the spectrum modifying effects of interior surface finishes for two reasons. Firstly, the spectral sensitivity of the photosensitive retinal ganglion cells most likely evolved in response to dominant environmental factors, and it is hypothesised that this could have been the varying spectral composition of daylight through the typical dawn to dusk diurnal cycle. Secondly, we wished to avoid results that were specific or at least in part determined by arbitrary choices of interior decor. The tracking of the various contributions from the daylight sources is described in Section 2.3. 


\subsection{Part B: Daylight transfer}

The daylight received at the eye was predicted using an 'in-house' lighting simulation research tool founded on the widely-used Radiance system [11]. The research tool has at its core a refined implementation of Tregenza's daylight coefficient approach [12]. The tool has been regularly updated and extended in response to challenges presented by various 'live projects', e.g. daylight modelling for the New York Times Building study [13].

The daylight coefficient approach requires that the sky be broken into many patches. The internal illuminance at a point that results from a patch of sky of known luminance is computed and cached. This is done for each patch of the hemispherical sky. It is then possible, in principle, to determine the internal illuminance for arbitrary sky/sun conditions using relatively simple (i.e. quick) arithmetic operations on matrices. The basic scheme described by Tregenza and Waters in 1983 was refined so that total illuminance arriving at a sensor point was computed as four separate components: direct sun $\left(\mathbf{E}^{s d}\right)$; indirect sun $\left(\mathbf{E}^{s i}\right)$; direct sky $\left(\mathbf{E}^{d}\right)$; and, indirect sky $\left(\mathbf{E}^{i}\right)$. Direct means arriving directly from the source, i.e. the sensor can "see" the source. Indirect means that the light arrived at the sensor after one or more reflections off surfaces inside or outside of the space. Thus the total daylight illumination $(\mathbf{E})$ is simply:

$$
\mathbf{E}=\mathbf{E}^{d}+\mathbf{E}^{i}+\mathbf{E}^{s d}+\mathbf{E}^{s i}
$$

Note the illuminance quantities are vectors containing all the values for an arbitrary number of sensor points or, alternatively, pixels in an image.

For the direct sky and indirect sky components, the sky vault was divided into 145 patches since this matched the resolution of the measured sky luminance patterns that were used to test and validate the approach [14]. Direct components of illumination are quick to compute - almost instantaneous. It is the indirect components that require computational effort since numerous inter-reflections need to be determined to ensure accurate results. Using the 145 patch scheme, separate daylight coefficients were computed for direct light $\left(\mathbf{D}^{d 145}\right)$ and indirect light $\left(\mathbf{D}^{i 145}\right)$. The daylight coefficient matrix for the direct sun component $\left(\mathbf{D}^{d 5 k}\right)$ was calculated using a finely-discretised set of approximately 5,000 points evenly distributed across the sky vault. This ensured that the displacement between any actually occurring sun position and that of the nearest pre-computed point was never greater than $2^{\circ}$ and typically of the order of $1^{\circ}$. This fine-scale discretisation for the sun points is commensurate with a time-step of approximately 5 minutes. Thus the progression of the direct sun illumination in the space will be accurately represented for time-steps down to a few minutes. The indirect light from the sun has, of course, much less of a strongly directional character than direct sun. Thus the indirect sunlight could be reliably determined using the daylight coefficient from a $\mathbf{D}^{i 145}$ patch that was nearby to the sun position. The indirect light from the sun was determined therefore from the nearest of the 145 indirect patches to the actually occurring sun position, i.e. $\mathbf{D}_{\beta}^{i 145}$. From validation tests on various discretisation schemes it was determined that this delivered high accuracy that was barely distinguishable from a four times higher resolution scheme [15]. Thus the individual illuminance components were determined from the various daylight coefficient matrices as follows:

$$
\begin{aligned}
\mathbf{E}^{d} & =\mathbf{D}^{d 145} \mathbf{c}^{145} \\
\mathbf{E}^{i} & =\mathbf{D}^{i 145} \mathbf{c}^{145} \\
\mathbf{E}^{\text {sd }} & =\mathbf{D}_{\beta}^{d 5 k} S^{\text {sun }} L^{\text {sun }} \\
\mathbf{E}^{\text {si }} & =\mathbf{D}_{\beta}^{i 145} S^{\text {sun }} L^{\text {sun }}
\end{aligned}
$$


Where $\mathbf{c}^{145}$ is the 145 element column vector which is the product of patch sky luminance and patch solid angle, and $S^{\text {sun }}$ and $L^{\text {sun }}$ are the solid angle and luminance of the sun respectively. A time-step of 15 minutes was determined to offer sufficient temporal resolution to capture the progression of daylight in the space. The hourly values in the climate files were interpolated to a 15 minute time step and the sun position and sky luminance patterns were determined at this time increment, i.e. approximately 17500 instantaneous daylight values throughout the year. More detail on this part of the model is given in the Appendix.

The scheme described above was used to predict the time-series of four components of (vertical) illuminance received at the eye for a period of a full year for a range of scenarios (described in Section 3).

\subsection{Part C: Implementation of the threshold-based photobiology model}

The photobiology-based model was described in detail in the Part 1 paper [8], with acknowledged limitations that are further considered in the Discussion section below. Much of the work carried out for that paper was to determine the parameters and calibration for three key features of the photobiology-based model: spectrum, intensity and timing. How each of these was implemented in the simulation is described below.

Prior to this work, climate-based daylight modelling had rarely considered the spectral properties of the two sources of daylight in the simulation - the sun and the hemispherical sky dome. The goal was usually to predict one or other of the climate-based illuminance metrics, e.g. useful daylight illuminance or daylight autonomy. Illuminance is of course defined with respect to the standard photopic curve $V(\lambda)$. The circadian sensitivity curve $C(\lambda)$ is believed to be similar in shape to the photopic curve, but shifted to the shorter (i.e. 'blue') wavelengths with the peak somewhere in the range 446 to $477 \mathrm{~nm}$ [16][17]. The circadian sensitivity curve used here is that derived in Pechacek et al [18]. The precise shape of the curve is still uncertain, and, in addition to dependencies on time of day [19], exposure duration [20] and prior photic history [21], it is likely that spectral sensitivity shifts occur after the onset of light exposure [22]. However these considerations should not affect the design of the framework described here. The proposed workflow is likely to undergo revisions as the data from photobiology experiments improves, especially in terms of adding dynamic spectral and time dependencies into the model [23][24].

The curve for circadian sensitivity used here is shown alongside that for the standard photopic sensitivity in Figure 3. Both curves are normalised to maximum equals 100. Also shown are the curves for the CIE standard daylight illuminants D55, D65 and D75, normalised so that the value for each at $555 \mathrm{~nm}$ equals 100 , i.e. the peak of the $V(\lambda)$ curve. Here we can see that, for any given (standard photopic) lux value, illuminant D75 has a greater 'circadian efficacy' than D65, which in turn has a greater 'circadian efficacy' than D55. We apply the following approximations: that solar beam radiation matches illuminant D55, overcast sky diffuse radiation matches illuminant D65 and that clear blue sky diffuse radiation matches illuminant D75.

It now becomes necessary to consistently employ 'circadian-equivalent' illuminance values in the tracking and summation of illuminance contributions from the various daylight sources. We chose to use D55 'circadian-equivalent' illuminance values (i.e. $E^{D 55}$ ), though the choice is arbitrary. The scaling factor $f_{75>55}$ to convert (photopic) lux values from a D75 illuminant to a D55 'circadian-equivalent' illuminance is determined from:

$$
f_{75>55}=\frac{\sum C(\lambda) D_{75}(\lambda)}{\sum C(\lambda) D_{55}(\lambda)} \cdot \frac{\sum V(\lambda) D_{55}(\lambda)}{\sum V(\lambda) D_{75}(\lambda)}
$$




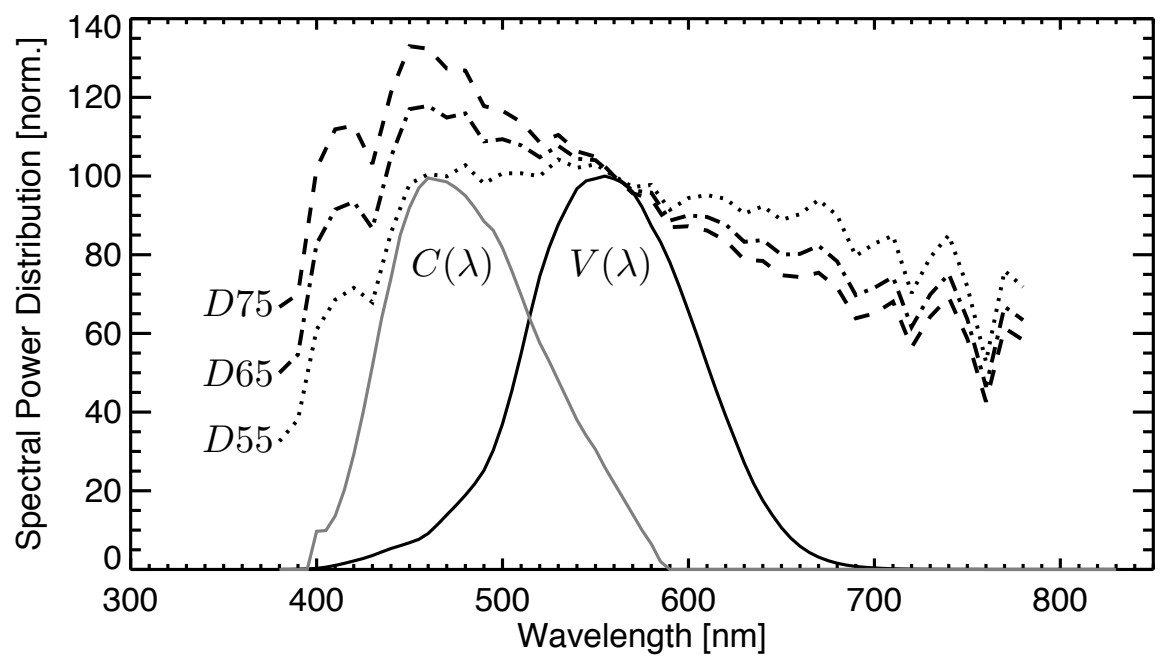

Figure 3: Spectral power distribution for CIE daylight illuminants associated to the three daylight sources alongside normalised photopic and circadian sensitivity curves $V(\lambda)$ and $C(\lambda)$ [reproduced from the Part 1 paper]

Each summation is carried out over all non-zero values for $V(\lambda)$ and $C(\lambda)$ respectively. Similarly, replace D75 with D65 in equation 3 to convert lux values from illuminant D65 to D55 'circadian-equivalent' illuminance. The factors to convert D65 and D75 to D55 'circadian-equivalent' illuminance were 1.10 and 1.16 respectively (see Part I paper [8]). The time-series for illuminance components received at the eye for direct and reflected skylight were thus converted to D55 'circadian-equivalent' illuminances using these factors depending on the estimated instantaneous sky condition, i.e. overcast (D65), clear (D75), or some partial blend of the two. Since light from direct and indirect sun was taken to match illuminant D55, there was no need to adjust these components. The four components were then summed to give a time-series for D55 'circadian-equivalent' illuminance. Thus the spectral character of the daylight sources was accounted for by tracking the (monochromatic) contributions of the various components rather than any explicit spectral modelling per se. Note the curves for the three D-illuminants do not need to be normalised a priori to give equal (photopic) lux, the $V(\lambda)$ summation terms in equation 3 correct for that.

The ramp-function described in Part 1 gave the likelihood of non-visual effect as a function of light received at the eye defined in terms of D55 equivalent illuminance. Below a minimum vertical illuminance at the eye of 210 lux (D55 equiv) the non-visual effect was 0\%. For an illuminances greater than 960 lux (D55 equiv) the effect was 100\%. A linear relation was assumed for values between these limits [8]:

$$
N_{V E}= \begin{cases}0, & \text { if } E^{D 55} \leq 210 \\ 100 E^{D 55} / 750-28, & \text { if } 210<E^{D 55}<960 \\ 100, & \text { if } E^{D 55} \geq 960\end{cases}
$$

This ramp-function was applied to the time-series of D55 'circadian-equivalent' illuminance to produce a time-series of percentage non-visual effect $N_{V E}$ for subsequent aggre- 
gation into annual cumulative values for each of the three time periods.

The photobiology model employs a fairly simple schema for timing. As mentioned above, this early stage of model development did not include factors such as duration, history, etc. Thus we considered only the instantaneous occurrence of a (percentage) non-visual effect and when it occurs in the day. For reasons explained in the Part I paper [8], we considered the day as having three distinct 'non-visual effect' periods:

- Early to mid-morning (6:00-10:00 h), where sufficient daylight illuminance can serve to phase advance the clock in the majority of people.

- Mid-morning to early evening (10:00 to 18:00 h), where high levels of daylight illuminance may lead to increased levels of subjective alertness without exerting substantial phase shifting effects on the clock.

- The rest as notional night-time (18:00 to 6:00 h), where daylight exposure that might trigger the non-visual effect is generally to be avoided.

As is the case with any daylighting measure - visual or non-visual - a 'snap-shot' value is usually of little worth. For task illuminance and general daylight availability, climatebased metrics such as useful daylight illuminance (UDI) and daylight autonomy (DA) have found favour with researchers and, increasingly, practitioners [25]. These metrics usually determine the spatial distribution (across horizontal planes) of the annual occurrence of daylight within certain specified ranges. The graphical methods devised to represent UDI and DA do not readily lend themselves for the visualisation of non-visual effects because now the view direction is one of the key factors determining the outcome, in addition to spatial position (i.e. proximity to glazing). A simple graphical device we refer to as the 'sombrero plot' was conceived to help visualise both the directional and spatial dimensions of simulated non-visual effect [8]. How this is employed in a practical simulation is described in the following section.

\subsection{Part D: Data visualisation}

At this early, speculative, stage in the modelling of non-visual effects we are interested primarily in an overview of the spatial distribution for prevailing non-visual effect in a space. Thus we determine a cumulative annual measure for non-visual effect based on the time-series of predicted values. The cumulative annual non-visual effect $C_{N V E}$ for each of the three non-visual effect periods $P$ is the sum of all the non-visual effect values occurring within that period divided by the total number of occurrences $n_{P}$ in the year of predicted values within that period:

$$
C_{N V E}^{P}=\frac{\sum_{t \in P} N_{V E}(t)}{n_{P}}
$$

Thus the cumulative annual non-visual effect is also normalised to a maximum of $100 \%$. For example, a cumulative annual value of, say, $40 \%$ could mean: an instantaneous $40 \%$ non-visual effect for $100 \%$ of the time; an instantaneous non-visual effect of $100 \%$ for $40 \%$ of the time (the remainder time having zero non-visual effect); or, as is evident from the temporal map of non-visual effect, something in-between these unlikely extremes. This is illustrated in Figure 4. The percentage instantaneous non-visual effect for a complete year (15 minute time-step) is shown using false-colour. To help readily identify the range limits - $0 \%$ and $100 \%$ - these have been shaded black and white respectively. Times when 
there was no daylight (i.e. where the global horizontal illuminance in the climate file was zero) are shaded grey. The horizontal green lines at hours 06:00, 10:00 and 18:00 delineate the three periods noted above. The cumulative annual non-visual effect for each of the three periods is shown in the 'sombrero plot' in Figure 4. The sombrero plot was devised to show any quantity that has a directional nature using a schema similar to the compass rose. The direction associated with the quantity is shown by the bisector of the two 'radii' that enclose the shaded area, the colour of which is used to indicate the magnitude of the quantity. For the study described here four possible view directions at $90^{\circ}$ increments were considered, i.e. same pattern as the cardinal compass points. Hence each annulus of the sombrero plot has four quadrants (one set of which are shown in Figure 4). The cumulative non-visual effect for the morning period is shown using the inner quadrant, the mid-morning to early evening period in the middle quadrant, and the notional night-time period in the outer quadrant (Figure 4). Note that the angle between the radii (e.g. $90^{\circ}$ ) has no bearing on the particular physical quantity other than to indicate a direction. In this case, a view direction for the calculation of vertical illuminance at the eye - which of course receives light from the visible hemisphere i.e. acceptance angle $180^{\circ}$ (rightmost graphic in Figure 4).

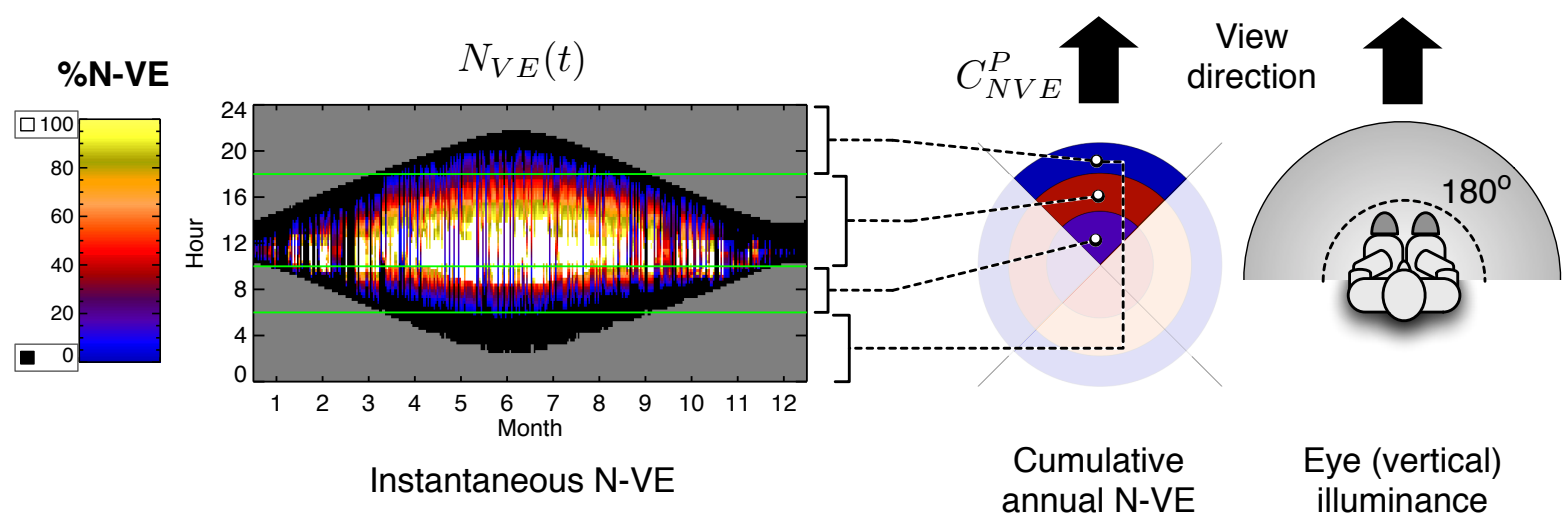

Figure 4: Instantaneous and cumulative annual non-visual effect (available in colour online)

\section{Application of the simulation model for $\mathrm{N}-\mathrm{VE}$}

The simulation model described above was applied to a residential building type for various climate scenarios as follows.

\subsection{Building scenario}

The residential dwelling was used as a 'virtual laboratory' to investigate the spatial distribution in predicted non-visual effect for various scenarios. The dwelling is based on a real house which has a design commonly found throughout Europe. Renderings of the 3D model for the residential building are shown in Figure 5. The sensitivity of predicted N-VE to daylight design interventions was investigated by predicting for cases with and without skylights - the images in Figure 5 show the building with skylights. The coloured areas in the plan view show the horizontal calculation planes where illuminance was predicted as part of an overall evaluation of daylight provision [26]. The spaces evaluated were: the living room (wg01); the kitchen (wg02); the entrance hall (wg03); small bathroom (wg04); 
large bathroom (wg05); and the stairs to the basement (wg06). Additionally, there are smaller square planes in three of the spaces: sixteen in the living room (wg01); four in the kitchen (wg02); and, one in the larger bathroom (wg05). These represent locations at head-height where vertical illuminance at the eye was predicted for the determination of non-visual effects. At each of these locations, the vertical illuminance was determined for four view directions, i.e. at $90^{\circ}$ increments. Due to space constraints only results for the living room (wg01) are presented in this paper.

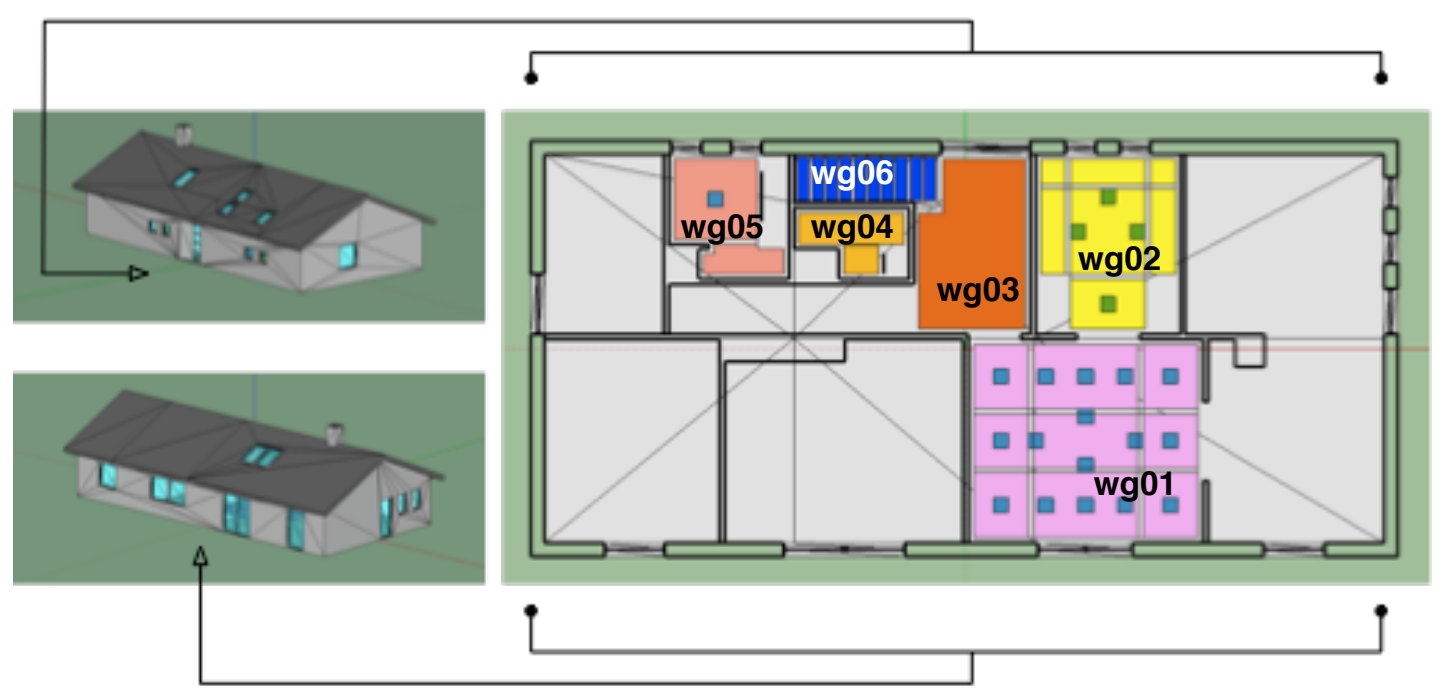

Figure 5: Images of the two main building facades (variant with skylights) together with a plan view showing the calculation planes for the spaces and the smaller, square planes for the N-VE model (available in colour online)

\subsection{Climate scenarios}

The building was evaluated for eight locales which cover a wide range of climate types with respect to daylight provision. The eight locales (assigned ID tag, city, country, lat/lon) are listed in Table 1. The climate data used for the simulations was diffuse horizontal illuminance and direct normal illuminance. The last column in Table 1 gives the number of "sunny" days for each of the climate files. There is no widely accepted definitive definition for the occurrence of a sunny day in a climate file. Here, a sunny day was taken to be one where more than half of the daily total of global horizontal illuminance was due to direct solar radiation. This quantity varied from 49 days (Moscow) to 194 (Madrid) and serves as a rough indicator to summarise the overall degree of "sunniness" for the climates. Additionally, the building was evaluated for four orientations under each climate.

\section{Results}

Using the simulation model described in the previous sections, the magnitude of the nonvisual effect produced by daylight illumination at the eye was predicted at a number of locations in the living room for the entire year at a 15 minute time-step, and for four horizontal view directions at $90^{\circ}$ increments. This was done for all 64 combinations of climate $(\times 8)$, building orientation $(\times 4)$ and building type $(\times 2)$. 


\begin{tabular}{lccrrr} 
ID & City/ & Country & Latitude & Longitude & $\begin{array}{r}\text { "Sunny" } \\
\text { days }\end{array}$ \\
\hline DEU-Hamburg & Hamburg & Germany & 53.63 & -10.00 & 50 \\
ESP-Madrid & Madrid & Spain & 40.41 & 3.68 & 194 \\
FRA-Paris & Paris & France & 48.73 & -2.4 & 64 \\
GBR-London & London & UK & 51.15 & 0.18 & 71 \\
ITA-Roma & Rome & Italy & 41.80 & -12.50 & 107 \\
POL-Warsaw & Warsaw & Poland & 52.17 & -20.97 & 53 \\
RUS-Moscow & Moscow & Russia & 55.75 & -37.63 & 49 \\
SWE-Ostersund & Ostersund & Sweden & 63.18 & -14.50 & 59
\end{tabular}

Table 1: The eight climate files used in the study

\subsection{N-VE at one location in a space}

Example output showing both the time-series (temporal maps) and cumulative occurrence (sombrero plot) for one location in the living room is given in Figure 6. The small inset graphic in the figure shows the position in the room of the calculation plane (red square) and the arrangement of the four temporal maps corresponds to the view directions illustrated by the arrows. Each temporal map is annotated with the view direction for this particular building orientation, i.e. ' $N$ ', ' $E$ ', ' $S$ ' and ' $W$ '. The shading is the same as that shown in Figure 4. The location selected for this example is at the back of the room away from the main window. As expected, the view towards the window (' $E$ ') shows the strongest N-VE in the associated temporal map. Next strongest overall is the view direction 'looking' towards the opposite wall ('N'), which will include a partial view of the window. The other two view directions show fairly weak N-VE in comparison. For the building orientation in this example, the main window was on an east-facing facade. Hence the morning period N-VE shows moderately strongly for those two views that include the window because of the ingress of morning sun. The cumulative annual $\mathrm{N}-\mathrm{VE}$ for each view direction for each of the three periods is shown in the corresponding quadrant sector of the sombrero plot (see Figure 4 and equation 5). The cumulative N-VE for the 'circadian' and 'alertness' periods for the view towards the window ('E') are both around 45 to $50 \%$ (shaded red - see legend). For other view directions the cumulative $\mathrm{N}-\mathrm{VE}$ is markedly smaller in magnitude.

\subsection{Visualisation of the spatial distribution of N-VE}

The spatial distribution in N-VE is visualised by placing a sombrero plot at each of the 16 sensor (i.e. head) locations where vertical illuminance at the eye was calculated. We shall not dwell on small numerical differences in predicted cumulative N-VE, rather our intention in this paper is to reveal, using graphical means, significant differences in cumulative N-VE due to design interventions such as the addition of a skylight. For example, in Figure 7 we can compare the predicted cumulative N-VE for the cases without and with skylights for the Ostersund (Sweden) climate (building orientation as indicated in the figure). For the case without skylights, the degree of N-VE is greatest for those view points/directions located closest to and directed towards the window - this is evident from the pattern of shading in the sombrero plots. The case with a skylight shows a markedly greater cumulative N-VE for all locations, and with less of a preference for those views directed towards the window. 


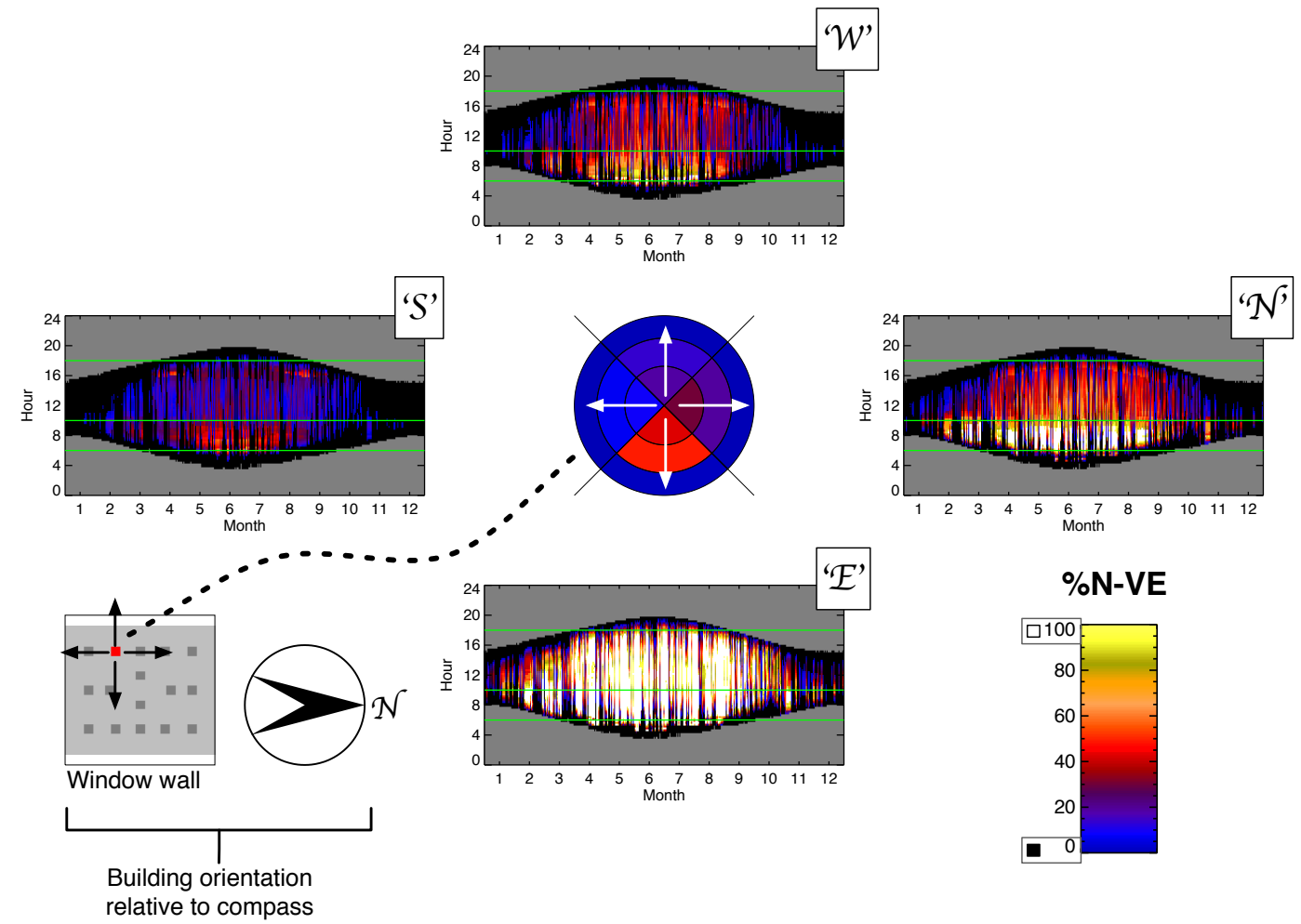

Figure 6: Example output showing four time-series (temporal maps) plots and cumulative occurrence (sombrero) plot for one point in the the living room (without skylight) for the Warsaw (Poland) climate file (available in colour online)

\subsection{N-VE summary for all combinations of climate and orientation}

We summarise the performance of the space in terms of the arithmetic mean value for the predicted non-visual effect across all 16 (eye-height) sensor locations and four view directions. This space-average cumulative annual N-VE was determined for the case with and without the skylight, and is plotted for all 32 combinations of climate and building orientation. The plotted points are grouped by location (e.g. DEU-Hamburg), and the building orientation relative to the compass is shown at the bottom of the plot - the order of the plotted points follows the order of the compass icons. The with-skylight point is marked by a small box, and a (vertical) line joining the with and without points indicates the change caused by the introduction of the skylight, Figure 8.

For any one climate locale there is often a factor two difference in predicted non-visual effect for the 'circadian period' across the four orientations, Figure 8. The preferential orientation with the highest 'circadian period' effect for any one climate locale is when the glazing faces east (i.e. compass legend $\ominus$ ). To maximise the non-visual effect for the 'alertness period', the preferred orientation is usually that with the glazing facing south (ه). Note that Madrid shows lower non-visual effect for the circadian period than perhaps one might expect given the high occurrence of clear skies. This is because Spain uses Central European Time and so, given its longitude, solar time is markedly later than clock time for much of the country. This and other locale-specific factors need to be borne in mind when making comparisons across countries. 


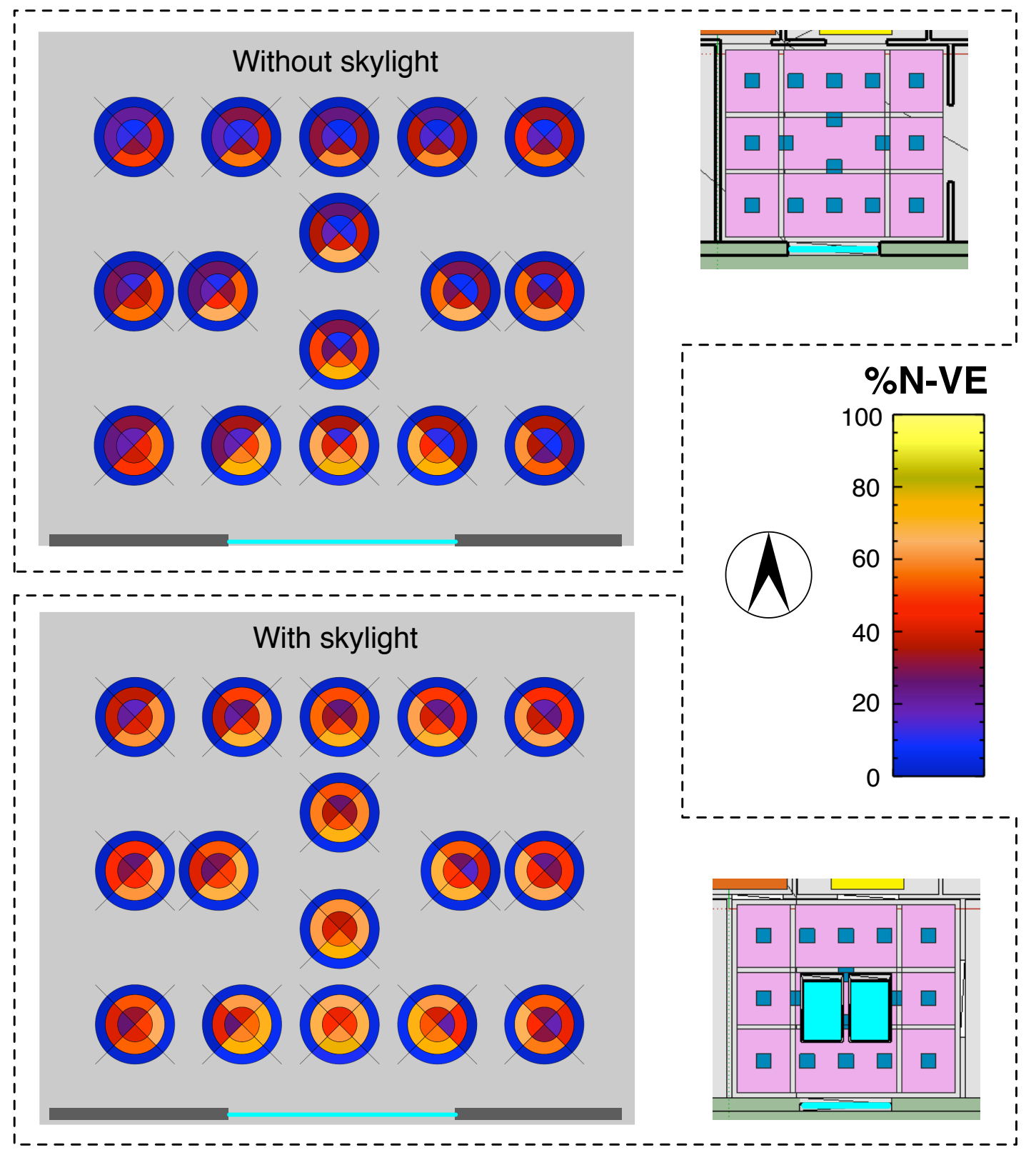

Figure 7: Predicted cumulative N-VE for case without and with skylights (Ostersund, Sweden) (available in colour online) 
Circadian period : 06h00-10h00
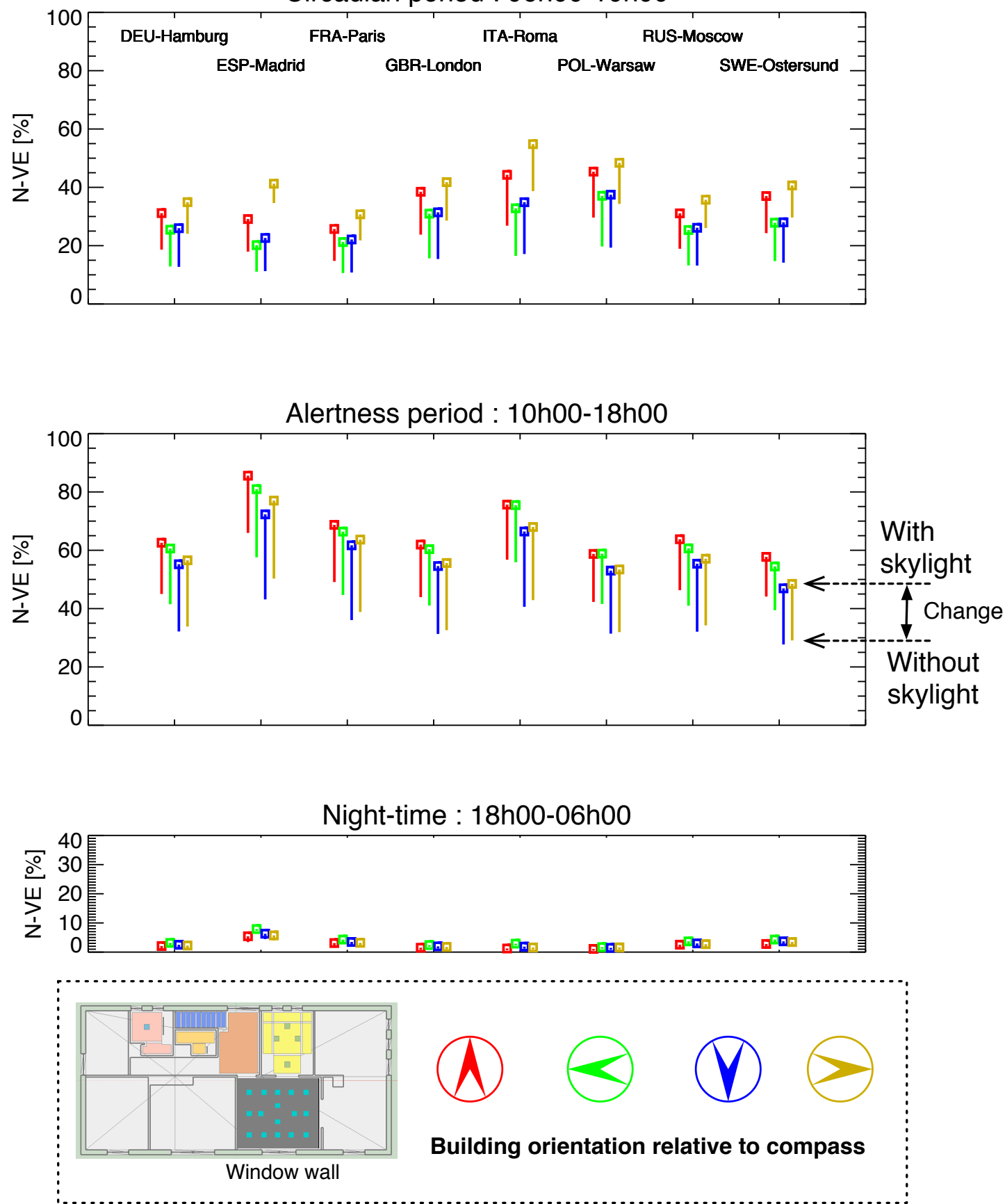

Figure 8: Cumulative non-visual effects for the living room (wg01) (available in colour online) 


\section{Discussion}

The climate-based simulation model and summary output from an exploratory study on the prediction and visualisation of non-visual effects for daylight in buildings has been described. The basis for the photobiology model was given in an earlier paper by Andersen, Mardaljevic \& Lockley [8]. While there is we believe sufficient empirical basis to begin the serious consideration of the evaluation of daylight in buildings for non-visual effects, it is clear that this model cannot be used as a predictor for non-visual effects in any absolute or deterministic sense because of its inherent limitations, as already described in the first paper [8]. Most notably, the model relies on instantaneous illuminance and on static exposure thresholds, and does not yet include dependancies on timing, duration and history of light exposure. In addition, and especially regarding circadian (phaseshifting) effects (compared to direct effects such as alertness), it is each individual's own sleep-wake cycle that will be the determining factor in what the actual influence of a specific light exposure pattern will be on this individual. Thus, predictions for nonvisual effects that rely exclusively on space-driven, instantaneous illuminance patterns are necessarily qualified by this limitation. That notwithstanding, such an approach remains a powerful way to establish the bases of a simulation framework ultimately able to make such predictions by integrating spectral and time-dependencies, as well as occupant profile in their analysis. A significantly enhanced photobiology model is in fact being developed by Andersen and co-workers for this purpose [23], including early investigations of how such a model could inform design when occupant profile and occupational dynamics are included [24].

The implementation of the static photobiology model on which the present paper relies [8] has resulted in a number of innovations that we hope will feature more widely in simulation research as the emerging field of predicted non-visual effects matures. The approximation of the spectral character of daylight through the separate tracking of sky and sun illuminance contributions, together with assumptions regarding 'blue' or 'grey' skies, introduced negligible computational overhead. The assumptions made are, we believe, commensurate with the starting point for the daylight sources, i.e. the integrated values in standardised climate files. The 'sombrero' graphical device is shown to be effective for communicating to the user complex spatio-temporal data relations in a manner that is almost intuitive - particularly so when comparing different glazing/facade configurations (Figure 7). The 'sombrero' plot lends itself to other representations of related quantities. For example, sectors of the graphic could be used to represent and compare categorical variables instead of view direction, e.g. varying levels of cumulative light exposure due to different shift-work patterns, Figure 9. For this, a 'personal' sombrero resulting from exposure to light at different locations could be compiled from various 'fixed' sombreros and a schedule to track the individual contributions. A hybrid deterministic/stochastic occupancy schedule could be devised to determine probable location and probable view direction as a function of time. This could of course include illumination received at the eye from artificial light sources, where the predicted non-visual effect would depend on the spectral character of the artificial light. To become a realistic prediction, the effect of timing (light adaptation, photic hostory) would, however, have to be accounted for, which would require a dynamic model to be developed, as mentioned at the end of this section.

As a visualisation tool, the existing framework could be adjusted to investigate photopic daylight exposure at the eye as a function of view direction and position for, say, residential or care homes, and possibly influence furniture layout and recommended occu- 
pancy schedule to maximise beneficial exposure to light. Similarly, the design of hospital wards could be evaluated in terms of daylight received at the eye by patients - here quite small changes in the angle of recline and orientation of the bed relative to the facade openings could result in large differences for prevailing exposure to daylight at the eye. More generally, the data visualisation schema devised for this study lends itself to a variety of other daylight applications where view direction in combination with timing are decisive factors.

As noted in the earlier paper and discussed above, we consider the model to be a preliminary framework for the investigation of daylight non-visual effects in buildings. Given the incomplete empirical data required for calibration, it would therefore be inappropriate at this stage to make any recommendations regarding exposure levels. Ongoing work carried out to revisit the framework, and refine the basis of the photobiological component, brings promising perspectives in starting to address the dynamics of light exposure and its non-visual effects by building upon empirical findings regarding intensity, spectrum, duration, history, and timing of exposure, all of which represent fields of investigation that are far from being exhaustively explored.

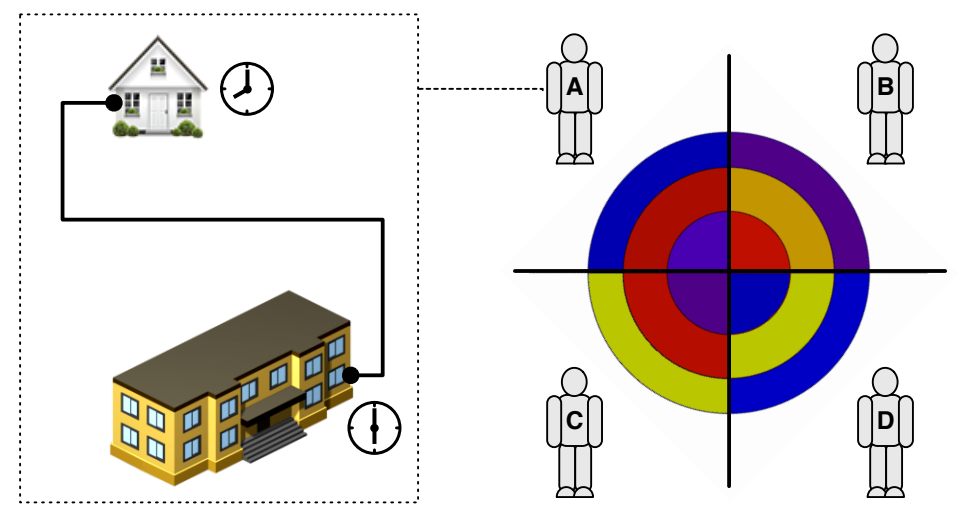

Figure 9: Sectors of the sombrero graphic used to represent and compare categorical variables, e.g. varying levels of cumulative light exposure due to different shift-work patterns - hypothetical example shown (available in colour online)

\section{Funding}

The research described in this paper is based a study commissioned by the VELUX Corporation, with institutional support from the Ecole Polytechnique Fédérale de Lausanne (M. Andersen) and Loughborough University (J. Mardaljevic).

\section{Acknowledgements}

The head image used in Figure 1 is courtesy of the NIGMS Image Gallery. Part of this work was carried out whilst Mardaljevic was at De Montfort University, Leicester, UK.

\section{References}

[1] S.W. Lockley and D. J. Dijk. Functional genomics of sleep and circadian rhythm. Journal of Applied Physiology, 92:852-862, 2002.

[2] L. Heschong. Daylighting and human performance. ASHRAE Journal, 44(6):65-67, 2002. 
[3] RG Foster, I Provencio, D Hudson, S Fiske, W De Grip, and M Menaker. Circadian photoreception in the retinally degenerate mouse ( $\mathrm{rd} / \mathrm{rd})$. Journal of comparative physiology. A, Sensory, neural, and behavioral physiology, 169(1), 071991.

[4] C. Czeisler and K. Wright. Influence of light on circadian rhythmicity. In F. W. Turek and P. C. Zee, editors, Neurobiology of sleep and circadian rhythms, pages 149-180. New York: M. Dekker, Inc, 1999.

[5] David M. Berson, Felice A. Dunn, and Motoharu Takao. Phototransduction by Retinal Ganglion Cells That Set the Circadian Clock. Science, 295(5557):1070-1073, February 2002.

[6] S. W. Lockley. Circadian Rhythms: Influence of Light in Humans, pages 971-988. Academic Press, Oxford, UK, 2009.

[7] Mark Rea, Mariana Figueiro, Andrew Bierman, and John Bullough. Circadian light. Journal of Circadian Rhythms, 8(1):1-10, December 2010.

[8] M. Andersen, J. Mardaljevic, and S. W. Lockley. A framework for predicting the non-visual effects of daylight - Part I: photobiology-based model. Lighting Research and Technology, 44(1):37-53, 032012.

[9] J. A. Clarke. Energy Simulation in Building Design 2nd Edition. ButterworthHeinemann, 2001.

[10] D. B. Crawley, L. K. Lawrie, F. C. Winkelmann, W. F. Buhl, Y. J. Huang, C. O. Pedersen, R. K. Strand, R. J. Liesen, Daniel E. Fisher, M. J. Witte, and J. Glazer. EnergyPlus: creating a new-generation building energy simulation program. Energy and Buildings, 33(4):319-331, 2001.

[11] G. Ward Larson and R. Shakespeare. Rendering with Radiance: The Art and Science of Lighting Visualization. San Francisco: Morgan Kaufmann, 1998.

[12] P. R. Tregenza and I. M. Waters. Daylight coefficients. Lighting Research and Technology, 15(2):65-71, 11983.

[13] E. S. Lee, S. E. Selkowitz, G. D. Hughes, R. D. Clear, G. Ward, J. Mardaljevic, J. Lai, M. N. Inanici, and V. Inkarojrit. Daylighting the New York Times headquarters building. Lawrence Berkeley National Laboratory. Final report LBNL-57602, 2005.

[14] J. Mardaljevic. The BRE-IDMP dataset: a new benchmark for the validation of illuminance prediction techniques. Lighting Research and Technology, 33(2):117-134, 2001.

[15] J. Mardaljevic. Daylight Simulation: Validation, Sky Models and Daylight Coefficients. PhD thesis, De Montfort University, Leicester, UK, 2000.

[16] K. Thapan, J. Arendt, and D. J. Skene. An action spectrum for melatonin suppression: evidence for a novel non-rod, non-cone photoreceptor system in humans. The Journal of physiology, 535(1):261-267, August 2001. 
[17] George C. Brainard, John P. Hanifin, Jeffrey M. Greeson, Brenda Byrne, Gena Glickman, Edward Gerner, and Mark D. Rollag. Action spectrum for melatonin regulation in humans: Evidence for a novel circadian photoreceptor. Journal of Neuroscience, 21(16):6405-6412, 2001.

[18] C. S. Pechacek, M. Andersen, and S. W. Lockley. Preliminary method for prospective analysis of the circadian efficacy of (day) light with applications to healthcare architecture. Leukos, 5(1):1-26, 2008.

[19] Melanie Rüger, Marijke C. Gordijn, Domien G. Beersma, Bonnie de Vries, and Serge Daan. Time-of-day-dependent effects of bright light exposure on human psychophysiology: comparison of daytime and nighttime exposure. American journal of physiology. Regulatory, integrative and comparative physiology, 290(5), May 2006.

[20] D. W. Rimmer, D. B. Boivin, T. L. Shanahan, R. E. Kronauer, J. F. Duffy, and C. A. Czeisler. Dynamic resetting of the human circadian pacemaker by intermittent bright light. American journal of physiology. Regulatory, integrative and comparative physiology, 279(5):R1574-9, November 2000.

[21] Kurt A. Smith, Martin W. Schoen, and Charles A. Czeisler. Adaptation of Human Pineal Melatonin Suppression by Recent Photic History. Journal of Clinical Endocrinology \&5 Metabolism, 89(7):3610-3614, July 2004.

[22] Joshua J. Gooley, Shantha M. W. Rajaratnam, George C. Brainard, Richard E. Kronauer, Charles A. Czeisler, and Steven W. Lockley. Spectral Responses of the Human Circadian System Depend on the Irradiance and Duration of Exposure to Light. Science Translational Medicine, 2(31):31-33, May 2010.

[23] M. L. Amundadottir, M. A. St. Hilaire, S. W. Lockley, and M. Andersen. Modeling non-visual responses to light: unifying spectral sensitivity and temporal characteristics in a single model structure. CIE Midterm conference - Towards a new century of Light, Paris, France, 15-16 April 2013.

[24] M. L. Amundadottir, M. Andersen, and S. W. Lockley. Proof-of-concept for the dynamic simulation of non-visual responses to daylight: application to the re-design of a healthcare facility. International Building Performance Simulation Association, Chambéry, France, 26-30 Aug 2013 (Submitted).

[25] C. F. Reinhart, J. Mardaljevic, and Z. Rogers. Dynamic daylight performance metrics for sustainable building design. Leukos, 3(1):7-31, 2006.

[26] J. Mardaljevic, M. Andersen, N. Roy, and J. Christoffersen. Daylighting metrics for residential buildings. CIE 27th Session, Sun City, South Africa, 2011.

[27] David Appelfeld, Andrew McNeil, and Svend Svendsen. An hourly based performance comparison of an integrated micro-structural perforated shading screen with standard shading systems. Energy and Buildings, 50(0):166-176, 72012.

[28] P. J. Littlefair. The luminous efficacy of daylight: a review. Lighting Research and Technology, 17(4):162-182, 121985.

[29] P. Tregenza. Analysing sky luminance scans to obtain frequency distributions of CIE Standard General Skies. Lighting Research and Technology, 36(4):271-279, 2004. 
[30] R. Perez, R. Seals, and J. Michalsky. All-weather model for sky luminance distribution-preliminary configuration and validation. Solar Energy, 50(3):235-245, 1993.

[31] J. Mardaljevic. Sky model blends for predicting internal illuminance: a comparison founded on the BRE-IDMP dataset. Journal of Building Performance Simulation, $1(3): 163-173,2008$.

[32] http://daysim.ning.com.

[33] http://www.diva-for-rhino.com.

[34] http://openstudio.nrel.gov.

\section{Appendix}

\section{Daylight coefficients}

The basic daylight coefficient 'engine' used for this study is described in detail in Mardaljevic's thesis [15]. A brief outline of the 'engine' noting the enhancements is provided in this Appendix. If $\Delta E_{\gamma \alpha}$ is the total illuminance produced at a point in a room by a small element of sky at altitude $\gamma$ and azimuth $\alpha$, then the daylight coefficient is defined as:

$$
D_{\gamma \alpha}=\frac{\Delta E_{\gamma \alpha}}{L_{\gamma \alpha} \Delta S_{\gamma \alpha}}
$$

where $L_{\gamma \alpha}$ is the luminance of the element of sky and $\Delta S_{\gamma \alpha}$ is the solid angle of the patch of sky [12]. The magnitude of the daylight coefficient $D_{\gamma \alpha}$ will depend on the physical characteristics of the room and the external environment, e.g. room geometry, surface reflectances, glazing transmissivity, outside obstructions and reflections etc. It is, however, independent of the distribution of luminance across the sky vault, since $\Delta E_{\gamma \alpha}$ varies in proportion to $L_{\gamma \alpha}$. The total illuminance $E$ produced at the point in the room is then calculated from:

$$
E=\int_{0}^{2 \pi} \int_{0}^{\pi / 2} D_{\gamma \alpha} L_{\gamma \alpha} \cos \gamma d \gamma d \alpha
$$

It is possible to determine a functional form for daylight coefficients (DCs) for idealised scenes such as an unobstructed horizontal surface [12]. However, some form of finite element calculation is needed for even the simplest realistic scene. If the sky were divided into $n$ angular zones, then for numerical evaluation, Eq 7 can be formulated as:

$$
E=\sum_{p=1}^{n} D_{p} S_{p} L_{p}
$$

This gives the illuminance as sum of $n$ products of $D, S$ and $L$, for each patch of sky $p$. The $n$ values of $D, S$ and $L$ can therefore be treated as vectors e.g. $\mathbf{D}=\left[D_{1}, D_{2}, \ldots, D_{n}\right]$. The formulation may be expanded to account for $m$ points in the room. The array of daylight coefficients then becomes a $m \times n$ matrix. The internal illuminances will then be described by a column vector E containing $m$ elements. Similarly, another column vector, c, can be formed from the $n$ products of angular size and luminance. This gives the compact matrix formulation:

$$
\mathrm{E}=\mathrm{D} \times \mathbf{c}
$$


The sky discretisation employed 145 patches. However, if implemented directly in a simulation, this patch scheme would often result in large errors for the computation of direct sun. As noted in Section 2.2, a much finer discretisation for the direct sun component was used to minimise this effect.

The method described in Mardaljevic's thesis to predict the direct contribution of a sky patch involved sending, from each calculation point, multiple rays distributed across a hemisphere and then tracking which rays intercepted with a particular patch of sky [15]. This approach was modified in 2007 to allow for the modelling of light redirecting glazing using the DC approach. Although not specifically required for the glazing types used for this study, the modified DC approach was used for the work described here. Note that, more recently, Radiance has been significantly enhanced to allow for the simulation of glazing systems with arbitrary bi-directional scattering distribution functions (BSDFs) within a generalised daylight coefficient framework [27]. This new approach is likely to become the standard DC implementation for Radiance in the future.

\section{Deriving sun and sky conditions from the climate data}

The luminance of the sun is derived directly from the value for direct normal illuminance in the climate, or, if illuminance values are not available, from the corresponding irradiance data via a luminous efficacy model [28]. The sun position may be given in the climate file or determined from the 'time-stamp' for that particular instant. The sky luminance pattern however must be inferred from the basic data in the climate file. There are a number of formulations that can be used to generate sky luminance patterns for a variety of conditions, e.g. overcast, clear, intermediate, etc. The first sky luminance pattern to be adopted by the CIE as a standard was the Moon and Spencer overcast sky formulation in 1955. A description for a standard clear sky pattern followed in 1973, and more recently we have seen the emergence of the CIE General Sky, which is a 'family' of 16 sky types covering the range from heavily overcast to very clear in fairly subtle gradations [29]. All of the CIE formulations are for a particular sky type, so it is the user who must select the sky type based on the (instantaneous) values read or derived from the climate file. In contrast to these, the Perez All-weather model automatically selects a pattern, e.g. heavily overcast, clear, etc., depending on the instantaneous values read from the climate file [30]. This convenience has resulted in the Perez All-Weather model being favoured for some studies, and also inclusion in some end-user daylight modelling tools. However, the Perez All-weather model has been shown to perform less reliably than a blend formulation which 'mixes' CIE overcast and clear sky patterns based on a simple rule [31]. This may be because the Perez All-weather model was founded on data collected only at one site, i.e. Berkeley (California, USA). Additionally, the model can occasionally produce distorted luminance patterns that contain spurious 'cusps' of negative luminance. For these reasons, the sky luminance patterns used for this study were derived from blends of CIE skies [31].

As noted in Section 2.3, the illumination from the sun, overcast sky and clear sky were assumed to correspond to, respectively, CIE standard illuminants D55, D65 and D75. It is acknowledged that this is a crude approximation. Whilst it might be possible in the future to improve on the parameterisation of this part of the model (e.g. a more suitable spectrum to approximate 'blue skies'), it is unlikely that refinements to include, say, the variation in spectrum across the sky vault would be worthwhile modelling. This is because uncertainties in, say, the spectral reflectance characteristics of external surfaces, obstructions, etc. are likely to overwhelm what are probably subtle effects in the overall model. 


\section{Annual simulations}

A consequence of the definition of the daylight coefficient is that, once computed, a single set of DC matrices can be used to quickly simulate internal illuminances for arbitrary climates and arbitrary building orientations. Thus the approach is well suited for parametric studies. The procedure is as follows:

- Load pre-computed daylight coefficients.

- Select climate file and assign a building orientation.

- For each time-step during daylight hours, generate the appropriate sky luminance pattern and sun description according to the values in the climate file and the orientation applied to the building.

- Derive internal illuminances from the pre-computed daylight coefficients and luminance patch values derived in the previous step.

- Process illuminance data applying the model for non-visual effects and generate summary metrics/graphics

\section{Climate-based daylight modelling (CBDM) software}

The software used to carry out CBDM simulations (employing Radiance as the 'engine' to predict daylight coefficients) is a bespoke research tool developed by Mardaljevic. It exists as a collection of scripts and procedures which are continually refined/updated in response to new challenges be they live projects or purely theoretical/research in nature. The software is a specialist tool with no graphical user interface, sparse documentation and continually changing. For these reasons it has not been released and is used solely 'in house', though the key algorithms etc. and various other details have been published.

End-user CBDM tools include DAYSIM [32], DIVA4RHINO [33] and OpenStudio [34] - all use Radiance as the lighting 'engine'. It is not known if any of these tools could be used directly or adapted/configured by the user for the climate-based modelling of non-visual effects. 